\title{
Welcome to the new Journal of Software Engineering Research and Development (JSERD)
}

\author{
Itana MS Gimenes ${ }^{1 *}$, Claudia Werner ${ }^{2}$ and Andre van der Hoek ${ }^{3,4}$
}

\author{
* Correspondence: \\ imsgimenes@uem.br \\ ${ }^{1}$ Departamento de Informática, \\ Universidade Estadual de Maringá \\ Av. Colombo, 5790, 87020-080 \\ Maringá, Paraná, Brazil \\ Full list of author information is \\ available at the end of the article
}

\section{Editorial}

Research is shared with the world through the publication of scientific papers in conferences and journals. Between these two, journals are the traditional means for archival publication of research results across a broad range of disciplines. So it is in software engineering. Despite the important role that our conferences serve in furthering our field, journals still serve the role of disseminating and archiving more complete and mature results. Providing this avenue is crucial, given how software is present in human life more than ever. As a result, our community has the great responsibility to invent, develop, and deploy new techniques that assist developers everywhere in creating high-quality, safe, and secure software through productive and economicallyfeasible processes. Only by 'seeing the research through', that is, pushing it further so we understand the full ramifications of our inventions, can we guarantee that what we propose actually helps make the world a better place. As such, journal publications are as important as ever.

One might question the need for another journal. After all, our community already has quite a few existing journals. Some of these, however, experience backlogs that lead to a long delay between the original submission and eventual publication. Others are specialized, focusing on one or at best a handful of specific subareas. JSERD emerged in this context as the result of extensive discussions in the Special Software Engineering Committee (CEES) of the Brazilian Computing Society (SBC) that lead to the recognition of the need for a broad software engineering journal that promotes rapid publication. JSERD is this journal, and is based on four major principles.

\section{JSERD is an Open Journal}

There have been many discussions on the social and human value of open journals within the broader discussions about open science, open data, and open education. The ultimate objective of these open movements is to disseminate information and knowledge around the world at no or little cost to the readers. Most research across the world has governmental support, which means public money, so it is fair that their results return to the public free as well. SpringerOpen provides authors a means of publishing open access papers while retaining copyright. Springer supports the submission, review, and access process to facilitate the work of the editorial board, reviewers, and authors. Authors (or their partner societies) pay for article processing fees, which are less than what is typically paid for conference participation or journal subscription.

(c) 2013 Gimenes et al.; licensee Springer. This is an Open Access article distributed under the terms of the Creative Commons Attribution License (http://creativecommons.org/licenses/by/2.0), which permits unrestricted use, distribution, and reproduction in any medium, provided the original work is properly cited. 


\section{JSERD is committed to high-quality papers}

Open does not necessarily mean low quality. To the contrary. We have assembled a strongly-committed editorial board of well-known software engineering researchers, who have been involved in selecting quality reviewers and ensuring the review process focuses on strong papers that report high-quality research. Authors of submitted papers have been receiving feedback that enables them to, sometimes significantly, improve their papers and respective research agendas.

\section{JSERD accepts different types of papers}

One of the innovations of JSERD is to offer authors the opportunity to publish four different types of papers, namely: (1) research articles, (2) industrial case studies, (3) reviews, and (4) software articles. This goes beyond existing software engineering journals, which generally publish research papers only. JSERD additionally provides a venue for well-described and well-analysed industrial case studies that lay important foundations rooted in real software engineering practice and experience, for systematic literature reviews, and for articles that describe software tools and explore the underlying design and implementation issues.

\section{JSERD offers a fast review process}

JSERD is particularly committed to providing a fast turn-around time on review and publication of submitted articles, without losing sight of the goal of publishing highquality papers. We work closely with the associate editors and reviewers to ensure timely reviews, as a result of which it has been possible to provide authors with feedback very quickly (on the order of 6 weeks). This allows authors to have their papers published in a timely manner befitting today's rapid pace of technological innovation.

These are the first articles of JSERD. It takes time to start a journal of high quality, as many authors tend to watch and see a journal reputation first before committing their papers to it. Our review process is rigorous and we are confident that JSERD's reputation will follow in time. SpringerOpen is working closely with Thomson Reuters (ISI) to ensure that citation analysis of articles published in JSERD will be available as soon as possible.

We hope you enjoy our journal, and will consider submitting an article in the near future.

\footnotetext{
Author details

'Departamento de Informática, Universidade Estadual de Maringá, Av. Colombo, 5790, 87020-080 Maringá, Paraná, Brazil. ${ }^{2}$ Programa de Engenharia de Sistemas e Computação, COPPE/UFRJ, Cidade Universitária, Rio de Janeiro 21941-972 Brazil. Donald Bren School of Information and Computer Sciences, University of California, Irvine, USA ${ }^{4}$ Department of Informatics, 5029 Donald Bren Hall, Irvine, CA 92697-3440 USA.
}

Received: 17 September 2013 Accepted: 20 September 2013

Published: 29 October 2013

doi:10.1186/2195-1721-1-1

Cite this article as: Gimenes et al: Welcome to the new Journal of Software Engineering Research and Development (JSERD). Journal of Software Engineering Research and Development 2013 1:1. 